\title{
Dynamic Time Series Reliability Analysis for Long-Life Mechanic Parts with Stress-Strength Correlated Interference Model
}

\author{
Bin Suo* \\ Institute of Electronic Engineering, China Academy of Engineering Physics, Mianyang, 621900, China
}

\begin{abstract}
Based on data of the equivalent stress from the ANSYS for a loaded hollow shaft, the correlation between a mechanical part's elastic modulus and the corresponding Von Mises stress is statistically verified in this paper. Using the Copula correlation theory, a static reliability model involving stress-strength interference is built. According to the performance degradation data of mechanical parts with long-life and high-reliability, deterministic time series models are used to extract the characteristic information of the distribution of degradation variables, and then a method is proposed for estimating the characteristic parameters of degradation strength and integrated stress. Two-stage maximum likelihood estimation is applied to determine the scalar degree of correlation between both, and then a reliability assessment of long-life mechanical parts is completed.
\end{abstract}

Keywords: stress-strength interference model; time series; correlation; copula; reliability; statistical analysis

(Submitted on October 5, 2018; Revised on November 21, 2018; Accepted on December 16, 2018)

(C) 2019 Totem Publisher, Inc. All rights reserved.

\section{Introduction}

In 1947, Freudenthal [1] proposed the famous stress-strength interference model, which formed the basis of quantitative analysis design of mechanical component reliability. In the stochastic probabilistic reliability analysis method, the stress and strength are usually assumed as static random variables that are independent of each other [2-7]. In practice, due to the longterm effects of mechanical parts under dynamic load, fatigue, wear, corrosion, aging, and other factors cause component strength monotone attenuation. On the other hand, due to crack initiation, deepening of corrosion degree, and changes of dimension gap, the stress value of the force point changes with time. In addition, it is possible to be subjected to random impact stress, which results in a random process rather than constant [8-11]. Therefore, the dynamic analysis of stressstrength double random process interference is more practical for the reliability design of mechanical parts.

The allowable load (comprehensive strength) for mechanical parts usually increases with the increase in size parameters of the parts, material physical properties, and good surface quality factor. Meanwhile, parts of the stress of the focus of variable (consolidation stress) change because of the same kinds of factors. Thus, the stress-strength of the parts is correlated. The reliability calculation of independent interference is only a simplified approximation of such characteristics, and the correlation failure is the universal characteristic of mechanical reliability engineering. It is also a key issue to be solved urgently [12-16]. The stress-strength correlation interference model considers the correlation and is more widely applied.

For high reliability and long-life products, using traditional life tests and even accelerated life tests is often difficult to obtain the full life span of the data samples within the given time, which causes the classical failure statistical methods to lose efficacy. Fatigue, corrosion, aging, and other degradation characteristics are typically presented in the failure process of high reliability and long-life products. During the test, the degradation parameters of this kind of products are tested, and the degraded data is obtained while the statistical analysis is also completed, which is an effective and cost-saving reliability evaluation method [17-24].

\footnotetext{
* Corresponding author.

E-mail address: suo.y.y@163.com
} 
In this paper, the finite element analysis software ANSYS is used to calculate the equivalent stress of a loaded hollow shaft, and the node Von Mises stress value is extracted to verify the correlation between the machinery of the hollow shaft Von Mises stress and parts comprehensive strength component (modulus of elasticity). Then, based on the Copula function, the reliability calculation model of stress-strength correlation is built. Lastly, with the performance degradation data of high long-life mechanical products, a deterministic trend combination time sequence model is adopted to give the reliability prediction method for the product reliability under the dynamic interference of stress-intensity correlation.

\section{Model Assumptions and Symbols}

For high reliability and long-life mechanical parts, the test results are obtained by testing the parameters (such as crack length, corrosion depth, abrasion degree, etc.) of the machine parts. Assuming that:

(1) $n$ samples were tested and the interval time was the same. The test time is denoted as $t_{0}, t_{1}, \cdots, t_{m}$.

(2) The comprehensive strength of the part under load time $t$ is $\{\delta(t), t \in T\}$. The density function of $\delta(t)$ is $f_{\delta_{t}}(\delta, \boldsymbol{\beta}(t))$, and the corresponding distribution function is $F_{\delta_{t}}(\delta, \boldsymbol{\beta}(t)) \cdot \boldsymbol{\beta}(t)$ is the distributed characteristic parameter, which in normal distribution is $\boldsymbol{\beta}(t)=\left(\mu_{\delta}(t), \sigma_{\delta}(t)\right)$.

(3) The combined stress of the parts is $\{S(t), t \in T\}$, in which the density function of $S(t)$ is denoted as $g_{s_{t}}(s, \boldsymbol{\alpha}(t))$, and the distribution function as $G_{s_{t}}(s, \boldsymbol{\alpha}(t)) . \boldsymbol{\alpha}(t)$ is the distributed characteristic parameter.

(4) The related structure of comprehensive strength $\delta$ and comprehensive stress $S$ is expressed by Copula $C_{\theta}(u, v)$, in which $\theta$ is the relative degree parameters of the two. The Gumbel Copula and the Clayton Copula are common positive/negative Copula families in engineering.

$$
\begin{gathered}
C^{\text {Gum }}(u, v ; \theta)=\exp \left(-\left[(-\ln u)^{\frac{1}{\theta}}+(-\ln v)^{\frac{1}{\theta}}\right]^{\theta}\right), \theta \in(0,1] \\
C^{C l a}(u, v ; \theta)=\max \left\{\left(u^{-\theta}+v^{-\theta}-1\right)^{-1 / \theta}, 0\right\}, \theta \in[-1,0)
\end{gathered}
$$

It can be proved by the limit theory and by Robida's law that when $\theta \rightarrow 1$, the variables described in $C^{\text {Gum }}$ tend to be independent of each other; when $\theta \rightarrow 0$, the variables described in $C^{G u m}$ tend to be perfect positive correlation; when $\theta \rightarrow-1, C^{C l a}$ reflects a completely negative correlation; and when $\theta \rightarrow 0, C^{C l a}$ tends to be independent.

(5) The performance test data of the $i^{\text {th }}$ test time $t_{j}$ is $x_{i}\left(t_{j}\right),(i=1,2, \cdots, n ; j=1,2, \cdots, m)$.

\section{Statistical Analysis of Correlation Interference Between Stress-Strength Components}

In this section, a large finite element analysis software ANSYS is applied for the stress calculation of mechanical parts. The model consists of a hollow shaft and a disk. The diameter of the disc is $200 \mathrm{~mm}$, the thickness is $25 \mathrm{~mm}$, the outside/inside diameter of the hollow shaft is $35 \mathrm{~mm} / 25 \mathrm{~mm}$, and the length is $150 \mathrm{~mm}$. The model adopts the SOLID45 unit, with a total of about 5040 units and 6720 nodes. The finite element model is shown in Figure 1. The hollow shaft and the disc are connected under an interference fit, and the interference amount is $0.04 \mathrm{~mm}$. The nonlinear analysis was calculated by using ANSYS, and the load was applied to the axial end with a weight of $120 \mathrm{~N}$. The materials used in the finite element calculation are CL60 steel, class B wheel steel, aluminum, and other 20 materials, as shown in Table 1.

The hollow shaft components of the disk are constructed by using the 20 materials in Table 1 . The disc section was extracted by ANSYS. As shown in Figure 2, the results of Von Mises stress at node 7 are shown in Table 2.

SPSS is utilized to analyze the elastic modulus of the material and the calculated Von Mises stress value. Moreover, the distribution was fitted and the characteristic parameter values were obtained. The statistical results of the characteristic parameters are shown in Table 3. 
Based on the comparison of normal distribution, uniform distribution, logarithmic normal, and exponential distribution, Table 4 shows the optimal approximate distribution of SPSS fitting.

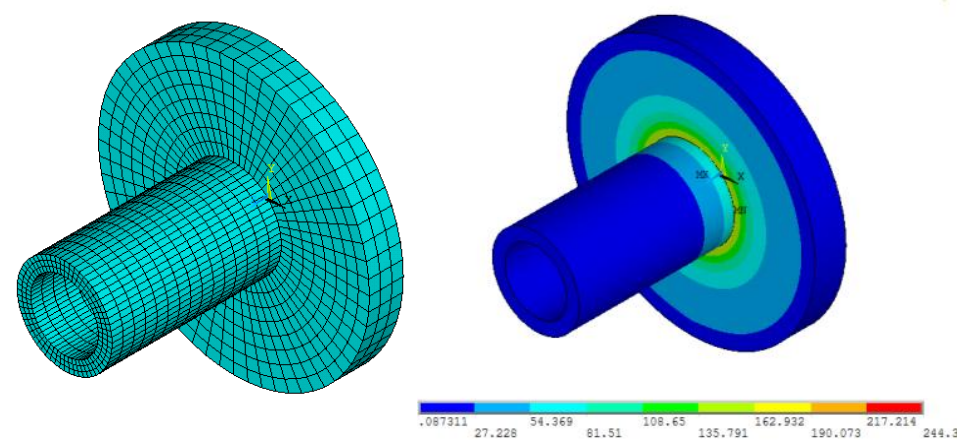

Figure 1. ANSYS computing model of hollow shaft and disk assembly

\begin{tabular}{|c|c|c|}
\multicolumn{4}{|c}{ Table 1. The elastic modulus of 20 materials used in the parts } \\
\hline No. & Material & Modulus of elasticity/GPa \\
\hline 1 & Cl60 steel & 205 \\
\hline 2 & B-wheel steel & 206 \\
\hline 3 & $45 \#$ steel & 71.7 \\
\hline 4 & Aluminum & 55 \\
\hline 5 & Porcelain & 17 \\
\hline 6 & Lead & 130 \\
\hline 7 & Gray cast iron & 105 \\
\hline 8 & Cast aluminum bronze & 95 \\
\hline 9 & Cold-drawing brass & 84 \\
\hline 10 & Rolling zinc & 100 \\
\hline 11 & Cast iron & 190 \\
\hline 12 & Stainless steel & 44.8 \\
\hline 13 & Magnesium & 207 \\
\hline 14 & Nickel & 46.2 \\
\hline 15 & Glass & 36.5 \\
\hline 16 & Graphite & 102.04 \\
\hline 17 & Titanium & 344.7 \\
\hline 18 & Tungsten & 11 \\
\hline 19 & Wood & 0.00784 \\
\hline 20 & Rubber & \\
\hline
\end{tabular}
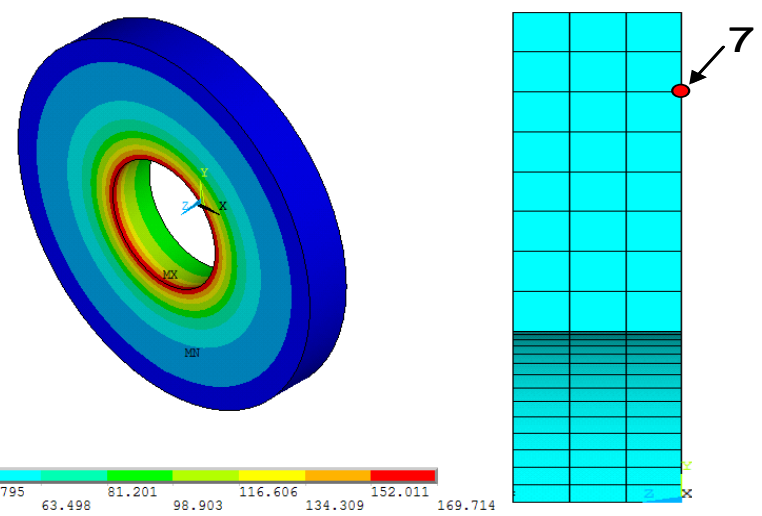

28.093
Figure 2. Finite element calculation of disk ANSYS 
Table 2. Elastic modulus of 20 materials used in the parts

\begin{tabular}{|c|c|c|}
\hline No. & Material & Von Mises/MPa \\
\hline 1 & Cl60 steel & 13.98746 \\
\hline 2 & B-wheel steel & 12.9309 \\
\hline 3 & $45 \#$ steel & 4.83837 \\
\hline 4 & Aluminum & 3.94067 \\
\hline 5 & Porcelain & 1.20912 \\
\hline 6 & Lead & 8.84996 \\
\hline 7 & Gray cast iron & 7.0457 \\
\hline 8 & Cast aluminum bronze & 6.22824 \\
\hline 9 & Cold-drawing brass & 5.66791 \\
\hline 10 & Rolling zinc & 6.62864 \\
\hline 11 & Cast iron & 12.95731 \\
\hline 12 & Stainless steel & 3.09795 \\
\hline 13 & Magnesium & 14.06966 \\
\hline 14 & Nickel & 3.08982 \\
\hline 15 & Glass & 2.5808 \\
\hline 16 & Graphite & 6.94558 \\
\hline 17 & Titanium & 23.39905 \\
\hline 18 & Tungsten & 0.75896 \\
\hline 19 & Wood & 0.00284 \\
\hline 20 & Rubber & \\
\hline & & \\
\hline
\end{tabular}

Table 3. The elastic modulus of 20 materials and the corresponding Von Mises stress distribution parameters

\begin{tabular}{|c|c|c|}
\hline & Modulus of elasticity & Von Mises stress \\
\hline $\mathrm{N}$ & 20 & 20 \\
\hline Mean & 112.15 & 7.59346 \\
\hline Std. Deviation & 87.665 & 5.94303 \\
\hline Minimum & 0 & 0.00284 \\
\hline Maximum & 345 & 23.39905 \\
\hline
\end{tabular}

Table 4. The elastic modulus of 20 materials and SPSS of corresponding Von Mises stress were fitted

\begin{tabular}{|c|c|c|c|}
\hline \multicolumn{2}{|c|}{ The fitting of exponential distribution } & Modulus of elasticity & Von Mises stress \\
\hline \multicolumn{2}{|c|}{$\mathrm{N}$} & 20 & 20 \\
\hline Exponential parameter & Mean & 112.15 & 7.5935 \\
\hline \multirow[t]{3}{*}{ Most extreme differences } & Absolute & .129 & .138 \\
\hline & Positive & .108 & .107 \\
\hline & Negative & -.129 & -.138 \\
\hline \multicolumn{2}{|c|}{ Kolmogorov-smirnov z } & .578 & .618 \\
\hline \multicolumn{2}{|c|}{ Asymp. Sig. (2-tailed) } & .892 & .840 \\
\hline
\end{tabular}

With the data of statistical samples in Tables 1 and 2, set the parts material elastic modulus for $X$ and the corresponding ANSYS to calculate the Von Mises stress for $Y$ to validate the mechanical parts of comprehensive strength, the modulus of elasticity for the intensity component, and the correlation between the parts under stress. The joint scatter of $X$ and $Y$, i.e., $\left(x_{i}, y_{i}\right),(i=1,2, \cdots, 20)$, is shown in Figure 3.

According to the correlation between material elastic modulus $X$ described in Figure 3 and the corresponding Von Mises stress $Y$, and combined with the fitting density function, $f_{X}(x), g_{Y}(y)$, shown in Table 4, the Gumbel copulas is used to build its related structure. It estimates the related degree of maximum likelihood for the result where $\hat{\theta} \approx 0.05$. The random simulated scatter diagram of copula $C^{\text {Gum }}(u, v ; \hat{\theta}=0.05)$ is shown in Figure 4. 


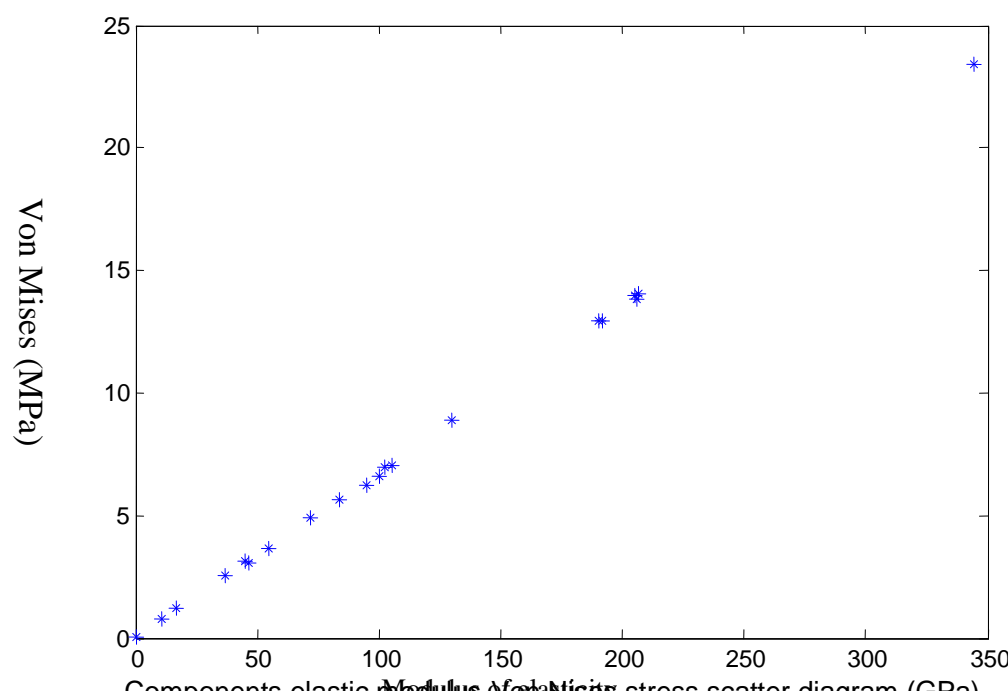

Components elastic modulus-dfohastisess stress scatter diagram (GPa)

Figure 3. Components elastic modulus -Von Mises stress scatter diagram

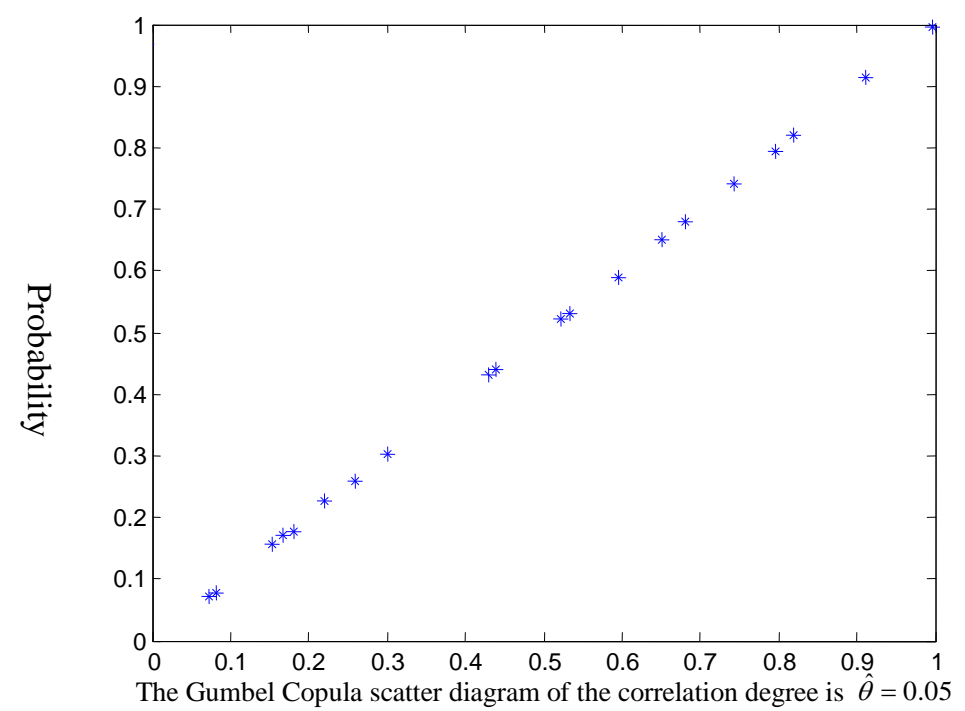

Figure 4. The Gumbel Copula scatter diagram of the correlation degree is $\hat{\theta}=0.05$

Similarly, the yield strength, tensile strength, shear strength, and other strength components of the 20 materials were analyzed and fitted to determine the density function of the component comprehensive strength $\delta(t)$ as $f_{\delta_{t}}(\delta$, $\boldsymbol{\beta}(t))$, and the comprehensive stress density function as $g_{s_{t}}(s, \boldsymbol{\alpha}(t))$. Also, the Copula selection and the correlation degree parameter estimation of $\hat{\theta}$ were completed.

\section{The Component Reliability Calculation Model based on the Stress-Strength Correlation}

In mechanical reliability design, the traditional stress-strength interference model assumes that the integrated stress $S$ and the comprehensive strength $\delta$ are independent of each other. The classical parts reliability prediction model is

$$
R=P(\delta>S)=\int_{-\infty}^{+\infty} g(s) \cdot \int_{s}^{+\infty} f(\delta) \mathrm{d} \delta \mathrm{d} s
$$

The correlation between $\delta$ and $S$ is presented that for an arbitrary $(x, y) \in R^{2}$,

$$
P(\delta>x, S \leq y) \geq P(\delta>x) P(S \leq y)
$$


The corresponding structure is characterized by Copula

$$
C_{\theta}\left(F_{\delta}(x), G_{S}(y)\right) \leq F_{\delta}(x) \cdot G_{S}(y)
$$

The Sklar theorem [25] guarantees the existence and uniqueness of the Copula $C_{\theta}$ in any two-dimensional random variable $(X, Y)$.

Theorem 1 [25] Let the marginal distribution function of the two-dimensional random variable be $F(\cdot), G(\cdot)$. Then, there exists only the two-dimensional Copula $C_{\theta}(u, v)$, which makes it arbitrary $x, y \in \bar{R}$,

$$
P(X \leq x, Y \leq y)=C_{\theta}(F(x), G(y))
$$

If the correlation between $X$ and $Y$ is not considered, this means they are independent of each other. Then,

$$
C_{\theta}(u, v)=u v
$$

In this situation, the reliability of the parts under stress $S$ and strength $\delta$ is

$$
\begin{aligned}
R & =P(\delta>S)=\int_{-\infty}^{+\infty} g(t) \cdot P(\delta>t \mid S=t) \mathrm{d} t \\
& =\int_{-\infty}^{+\infty} g(t) \cdot\left[\int_{t}^{+\infty} f_{\delta \mid S=t}(\delta \mid s) \mathrm{d} \delta\right] \mathrm{d} t \\
& =\int_{-\infty}^{+\infty} g(s) \cdot\left[\left.\int_{s}^{+\infty} \frac{\partial^{2} C_{\theta}(u, v)}{\partial u \partial v} \cdot f(\delta)\right|_{\substack{u=F(\delta) \\
v=G(s)}} \mathrm{d} \delta\right] \mathrm{d} s
\end{aligned}
$$

In the situation that the integrated stress and comprehensive strength of the parts are independent, $\frac{\partial^{2} C_{\theta}(u, v)}{\partial u \partial v}=1$, and then

$$
R=P(\delta>S)=\int_{-\infty}^{+\infty} g(s) \cdot \int_{s}^{+\infty} f(\delta) \mathrm{d} \delta \mathrm{d} s
$$

The results are consistent with the classical independent interference reliability calculation model. In fact, an independent relationship is only a special case of correlation, and the independent interference model is only a simplified special case of the correlation interference model.

\section{Dynamic Timing Reliability Statistical Analysis of Long-Life Mechanical Parts}

\subsection{Dynamic Timing Estimation of Characteristic Parameters of Degradation Strength Distribution}

According to the performance degradation data $x_{i}\left(t_{j}\right)$ samples of $t_{j}$ at the time of detection, the dynamic strength samples, $\delta_{i}\left(t_{0}\right), \delta_{i}\left(t_{1}\right), \cdots, \delta_{i}\left(t_{m}\right)$, of the parts are transformed based on the method of strength determination [26-27]. The distribution type $f_{\delta_{t}}(\delta)$ is determined by non-parametric distribution fitting. In most working conditions, the normal distribution is the most common situation for the random diversity of the interfering factors, so the normal distribution is chosen to be related.

The estimation of distribution characteristic parameters $\boldsymbol{\beta}(t)$ is realized with maximum likelihood estimation:

$$
\begin{aligned}
L\left(\boldsymbol{\beta}\left(t_{j}\right)\right) & =L\left(\mu_{\delta}\left(t_{j}\right), \sigma_{\delta}\left(t_{j}\right)\right) \\
& =\prod_{i=1}^{n} f_{\delta_{t}}\left(\delta_{i}\left(t_{j}\right), \mu_{\delta_{t}}\left(t_{j}\right), \sigma_{\delta_{t}}\left(t_{j}\right)\right)
\end{aligned}
$$


By solving the likelihood equations, we get the parameter vector estimation sequence of $\left(t=t_{0}, t_{1}, \cdots, t_{m}\right)$ and the moment component strength $\hat{\boldsymbol{\beta}}(t)=\left(\hat{\mu}_{\delta}(t), \hat{\sigma}_{\delta}(t)\right)$, in which

$$
\left\{\hat{\mu}_{\delta}(t)\right\}=\left\{\hat{\mu}_{\delta}\left(t_{0}\right), \hat{\mu}_{\delta}\left(t_{1}\right), \cdots, \hat{\mu}_{\delta}\left(t_{m}\right)\right\},\left\{\hat{\sigma}_{\delta}(t)\right\}=\left\{\hat{\sigma}_{\delta}\left(t_{0}\right), \hat{\sigma}_{\delta}\left(t_{1}\right), \cdots, \hat{\sigma}_{\delta}\left(t_{m}\right)\right\}
$$

Due to the long-term dynamic load of mechanical parts, its strength $\{\delta(t)\}$ is attenuated randomly. The dynamic components $\left\{\hat{\mu}_{\delta}(t)\right\}$ and $\left\{\hat{\sigma}_{\delta}(t)\right\}$ of the characteristic parameter vector $\hat{\boldsymbol{\beta}}(t)$ show the deterministic trend sequence. For this purpose, the dynamic effect of the fitting intensity parameters of the quadratic mean trend model is established:

$$
\left\{\begin{array}{l}
\hat{\mu}_{\delta}(t)=c_{0}+c_{1} t+c_{2} t^{2}+\mu(t) \\
\hat{\sigma}_{\delta}(t)=d_{0}+d_{1} t+d_{2} t^{2}+\sigma(t)
\end{array}\right.
$$

Where $c_{i}, d_{i},(i=0,1,2)$ is the parameter of undetermined trend, which can be processed by standard regression analysis. First, the concrete form is fitted, namely, based on the least squares estimation method. With the empirical samples $\left\{\hat{\mu}_{\delta}\left(t_{0}\right), \hat{\mu}_{\delta}\left(t_{1}\right), \cdots, \hat{\mu}_{\delta}\left(t_{m}\right)\right\}$ and $\left\{\hat{\sigma}_{\delta}\left(t_{0}\right), \hat{\sigma}_{\delta}\left(t_{1}\right), \cdots, \hat{\sigma}_{\delta}\left(t_{m}\right)\right\}$, the determination of the estimated value $\hat{c}_{i}, \hat{d}_{i}$ is completed. $\mu(t)$ and $\sigma(t)$ are the wide stationary process of the zero mean, and then the residual $\left\{\hat{\mu}_{\delta}(t)-\mu(t)\right\}$ and $\left\{\hat{\sigma}_{\delta}(t)-\sigma(t)\right\}$ are analyzed and modeled in the stationary process. Through the model (12), the dynamic prediction of the characteristic parameters $\hat{\mu}_{\delta}\left(t_{d}\right)$ and $\hat{\sigma}_{\delta}\left(t_{d}\right)$ of the degradation intensity $\left\{\delta\left(t_{d}\right)\right\}$ of the high long-life machine parts in the future $t_{d}$ is completed. Thus, the distribution density function $f_{\delta_{t_{d}}}\left(\delta, \boldsymbol{\beta}\left(t_{d}\right)\right)$ of the component strength in $t_{d}$ time is determined.

For the dynamic distribution density function $g_{s_{t}}(s, \boldsymbol{\alpha}(t))$ of the time stress course of the parts under the load, the same can be obtained according to the above time $\{S(t)\}$ sequence statistical methods, which will not be repeated here.

\subsection{Calculation of Dynamic Coherence Reliability of Stress-Strength and Estimation of Related Degree Parameters}

Combined with stress-strength interference correlation of the static reliability calculation model (9) and the above section of part stress, a statistical method to determine the dynamic distribution of intensity and then the high long-life part time dynamic reliability model is expected in the future time $t_{d}$.

$$
\begin{aligned}
R\left(t_{d}\right) & =P\left(\hat{\delta}\left(t_{d}\right)>\hat{S}\left(t_{d}\right)\right) \\
& =\int_{-\infty}^{+\infty} g_{s_{t_{d}}}\left(s, \hat{\boldsymbol{\alpha}}\left(t_{d}\right)\right) \cdot\left[\left.\int_{s}^{+\infty} \frac{\partial^{2} C_{\theta_{t d}}(u, v)}{\partial u \partial v} \cdot f_{\delta_{t_{d}}}\left(\delta, \hat{\boldsymbol{\beta}}\left(t_{d}\right)\right)\right|_{\substack{u=F_{\delta_{t_{d}}}\left(\delta, \hat{\boldsymbol{\beta}}\left(t_{d}\right)\right) \\
v=G_{s_{d}}\left(s, \hat{\mathbf{\alpha}}\left(t_{d}\right)\right)}} \mathrm{d} \delta\right] \mathrm{d} s
\end{aligned}
$$

For the determination of the correlation degree $\theta_{t_{d}}$ of the components' comprehensive stress process $\left\{S\left(t_{d}\right)\right\}$ and the degradation intensity $\left\{\delta\left(t_{d}\right)\right\}$ in the correlation interference model, it can be based on the empirical sample $\delta_{i}\left(t_{j}\right), s_{i}\left(t_{j}\right)$, $(i=1,2, \cdots, n ; j=1,2, \cdots, m)$, and the second phase maximum likelihood estimation obtains $\hat{\theta}_{t_{j}}$.

$$
L\left(\theta_{t_{j}}\right)=\prod_{i=1}^{n}\left[c_{\theta_{t_{j}}}\left(u_{1}, u_{2}, \cdots, u_{p}\right) \cdot \prod_{k=1}^{p} f_{X_{k}}\left(x_{i k}\left(t_{j}\right), \hat{\mu}_{X_{k}}\left(t_{j}\right), \hat{\sigma}_{X_{k}}\left(t_{j}\right)\right)\right]
$$

Where $c_{\theta_{t_{j}}}$ and $C_{\theta_{t_{j}}}$ are the density function, i.e., $c_{\theta_{t_{j}}}=\left.\frac{\partial C_{\theta_{t_{j}}}\left(u_{1}, u_{2}, \cdots, u_{p}\right)}{\partial u_{1} \partial u_{2} \cdots \partial u_{n}}\right|_{u_{k}=F_{X_{k}}\left(x_{i k}\left(t_{j}\right) \hat{\mu}_{X_{k}}\left(t_{j}\right), \hat{\sigma}_{X_{k}}\left(t_{j}\right)\right)}$. 
In most conditions, due to the size of the parts abrasion, poor surface quality, aging, corrosion, fatigue, and other reasons, the stress-strength interference of A related degree showed $\theta_{t}$ trend of average unabated by considering the effect of dynamic load. The exponential smoothing method is used to predict the timing sequence $\left\{\hat{\theta}_{t_{j}}\right\}$, and $\hat{\theta}_{t_{d}}$ is obtained.

$$
\hat{\theta}_{t_{j}}=a \hat{\theta}_{t_{j}}+(1-a) \hat{\theta}_{t_{j-1}}
$$

Where $0<a<1$ is the smoothing coefficient, whose value is determined by the least square regression of the sample sequence and in most cases satisfies $0.05<a<0.3$.

\section{Examples}

There is a linkage mechanism in a certain machine, which is under tension when working. The tension follows the normal distribution, where the mean value and standard deviation are $\bar{F}=35 \mathrm{kN}$ and $\sigma_{F}=3.66 \mathrm{kN}$ respectively. The material of the linkage mechanism is $\mathrm{A}_{5}$ steel, and its tensile strength follows the normal distribution, where the mean value and standard deviation are 50MPa and 4MPa respectively. The cross-section area of the linkage mechanism is $896 \mathrm{~mm}^{2}$. At this situation, we need to analyze the reliability of the linkage mechanism.

The mean and standard deviation of tensile stress of the mechanical linkage are:

$$
\begin{gathered}
\bar{S}=\frac{\bar{F}}{A}=\frac{35 \times 10^{3} \mathrm{~N}}{896 \times 10^{-6} \mathrm{~m}^{2}}=39.1 \mathrm{MPa} \\
\sigma_{S}=\frac{\sigma_{F}}{A}=4.08 \mathrm{MPa}
\end{gathered}
$$

The correlation structure between tensile stress $S$ and tensile strength $\delta$ conforms to the Clayton model $C_{\theta}(u, v)=\left(u^{-\theta}+v^{-\theta}-1\right)^{-1 / \theta}$. The probability density function and cumulative probability distribution of $S$ are:

$$
\begin{gathered}
g(s)=\frac{1}{\sqrt{2 \pi} \times 4.08} \exp \left(-\frac{(s-39.1)^{2}}{2 \times 4.08^{2}}\right) \\
u=G(s)=\int_{0}^{s} g(x) \mathrm{d} x
\end{gathered}
$$

The probability density function and cumulative probability distribution of tensile strength $\delta$ are:

$$
\begin{gathered}
f(\delta)=\frac{1}{\sqrt{2 \pi} \times 4} \exp \left(-\frac{(\delta-50)^{2}}{2 \times 4^{2}}\right) \\
v=F(\delta)=\int_{0}^{\delta} f(x) \mathrm{d} x
\end{gathered}
$$

Then, by substituting the above two formulas into the stress-strength interference reliability model, the initial reliability $R$ of the mechanical linkage is obtained with the change of the correlation degree parameters $\theta$, as shown in Figure 5 .

From Figure 5, it can be seen that the link reliability calculated by stress $S$ and strength $\delta$ correlation interference is between [0.9064,0.9719], and it is a continuous value changing with the correlation degree parameter $\theta$.

$R=0.9064$ at the left end of the interval is the reliability value when the stress $S$ and the strength $\delta$ are completely inversely correlated linear functions $(\theta=-1)$. If the traditional interference model (10) is adopted according to the independence theory, the reliability is calculated as the right endpoint value of the interval, $R=0.9719$. 
Obviously, the traditional interference model supposes that stress and strength are independent, which tends to open up the calculation results and may lead to overestimation of product reliability.

If the Monte Carlo method is adopted to simulate 4000 times by computer, the simulated sample value is substituted into the maximum likelihood estimation Equation (13), and the correlation degree parameter is obtained as -0.3517 . At this point, the correlation structure of stress $S$ and strength $\delta$ is shown in Figure 6, and the reliability of the mechanical linkage can be obtained from (8) of $R=0.9512$.

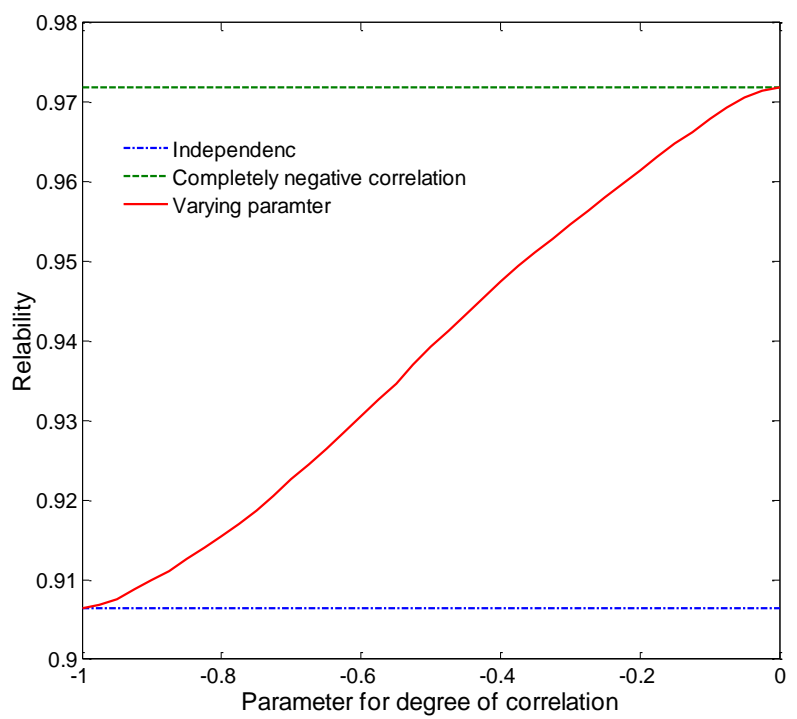

Figure 5. Time-varying reliability of the mechanical linkage

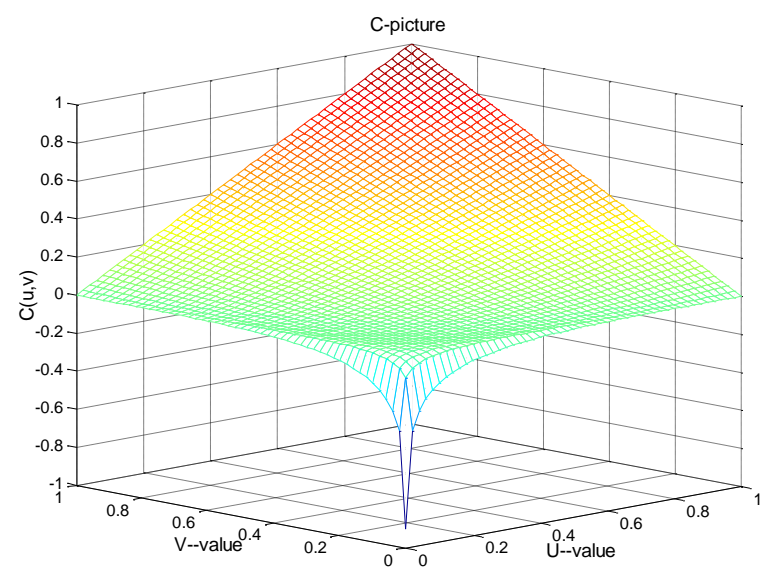

Figure 6. Stress-strength related structures Clayton copula model when $\theta=-0.3514$

\section{Conclusions}

The analysis of correlation between stress and strength is the theoretical basis of reliability calculation of mechanical parts Based on this, the reliability calculation model of stress-strength correlation interference is a generalization of the classical simplified model and becomes more suitable for practical applications.

The statistical theory of time series is an effective method for reliability analysis of long-life products. Based on the long-life and high mechanical parts performance degradation data using the deterministic trends combined time-series model, the comprehensive stress process of parts dynamic degradation characteristics parameter estimation method can be given. Based on two-stage maximum likelihood estimation, the comprehensive stress and comprehensive strength correlation degree are determined to complete the reliability evaluation of the stress-intensity correlation interference of long-lived parts. However, parameter estimation, correlation structure determination, and distribution prediction are all dependent on statistical analysis and information extraction of performance detection data. In general, the larger the sample size is, the more accurate the calculation results will be. Moreover, compared with the classical simplified model, this model is more computationally intensive. 


\section{References}

1. Y. M. Liu, Y. M. Shi, and B. Liu, "Stress-Strength Reliability Analysis of System with Multiple Types of Components using Survival Signature," Journal of Computational and Applied Mathematics, Vol. 342, No. 1, pp. 375-398, 2018

2. A. O. Connor and A. Mosleh, "A General Cause based Methodology for Analysis of Common Cause and Dependent Failures in System Risk and Reliability Assessments," Reliability Engineering \& System Safety, Vol. 145, No. 2, pp. 341-350, 2017

3. A. M. Freudenthal, "The Safety of Structures," Transaction on ASCE, Vol. 112, pp. 125-159, 1997

4. G. Levitin, "In Corporation Common-Cause Failures into No Repairable Multi-State-Parallel System Analysis," IEEE Transactions on Reliability, Vol. 50, No. 4, pp. 380-388, 2001

5. L. Li, M. Liu, W. M. Shen, and G. Q. Cheng, "An Expert Knowledge-based Dynamic Maintenance Task Assignment Model using Discrete Stress-Strength Interference Theory,” Knowledge-based Systems, Vol. 131, No. 4, pp. 135-148, 2017

6. W. Peng, Y. F. Li, Y. J. Yang, J. Mi, and H. Z. Huang, "Bayesian Degradation Analysis with Inverse Gaussian Process Models under Time-Varying Degradation Rates," IEEE Transactions on Reliability, Vol. 99, No. 7, pp. 1-13, 2017

7. L. Y. Xie, J. Y. Zhou, and C. Z. Hao, "System Level Load-Strength Interference based Reliability Modeling of k-out-of $n$ system," Reliability Engineering and System Safety, Vol. 84, No. 3, pp. 311-317, 2004

8. Z. W. An and H. Z. Huang, "A Discrete Stress Strength Interference Model based on Universal Generating Function," Reliability Engineering and System Safety, Vol. 93, No. 1, pp. 1485-1490, 2008

9. Z. H. Chen and S. R. Zheng, "Life Distribution based Degradation Analysis," IEEE Transactions on Reliability, Vol. 54, No. 1, pp. 3-10, 2005

10. H. Y. Dui, S. B. Si, and C. M. Richard, "Importance Measures for Optimal Structure in Linear Consecutive-k-out-of-n Systems," Reliability Engineering \& System Safety, Vol. 169, No. 1, pp. 339-350, 2017

11. W. Huang and D. L. Dietrich, "An Alternative Reliability Modeling Approach using Maximum Likehood Estimation," IEEE Transactions on Reliability, Vol. 54, No. 2, pp. 310-317, 2005

12. C. Diallo, U. Venkatadri, A. Khatab, and Z. J. Li, "Optimal Selective Maintenance Decisions for Large Serial k-out-of-n: G Systems under Imperfect Maintenance”, Reliability Engineering \& System Safety, Vol. 175, No. 1, pp. 234-245, 2018

13. J. Leander, "Reliability Evaluation of the Eurocode Model for Fatigue Assessment of Steel Bridges," Journal of Constructional Steel Research, Vol. 141, No. 5, pp. 1-8, 2018

14. Y. M. Liu, Y. M. Shi, X. C. Bai, and B. Liu, "Stress-Strength Reliability Analysis of Multi-State System based on Generalized Survival Signature," Journal of Computational and Applied Mathematics, Vol. 342, No. 1, pp. 274-291, 2018

15. Q. Sun, J. Y. Zhao, and J. L. Zhou, "Stress-Strength Interference Reliability Analysis Considering Stochastic Multi-Stress and Strength Aging Degradation," Chinese Journal of Computational Mechanics, Vol. 24, No. 3, pp. 358-361, 2007

16. Z. Yang, D. Zhu, C. Chen, H. Tian, and J. Guo, "Reliability Modelling of CNC Machine Tools based on the Improved Maximum Likelihood Estimation Method," Mathematical Problems in Engineering, Vol. 2018, No. 4, pp. 1-11, 2018

17. H. X. Guo, B. Suo, and G. Q. Zhang, "Reliability Bounds based on Universal Generating Function and Discrete Stress-Strength Interference Model," International Journal of Plant Engineering and Management, Vol. 35, No. 3, pp. 175-187, 2017

18. M. M. Hu, P. Wei, and J. J. Tang, "Fatigue Reliability Evaluation Model based on Residual Strength Tests of Whole Damage Region," Journal of Mechanical Strength, Vol. 25, No. 1, pp. 102-104, 2003

19. V. M. Hoepfer, J. H. Saleh, and K. B. Marais, "On the Value of Redundancy Subject to Common-Cause Failures: Toward the Resolution of an on-Going Debate”, Reliability Engineering \& System Safety, Vol. 94, No. 12, pp. 1904-1916, 2017

20. G. Jin and D. Matthews, "Reliability Demonstration for Long-Life Products based on Degradation Testing and a Wiener Process Model," IEEE Transactions on Reliability, Vol. 63, No. 3, pp. 781-797, 2014

21. M. Rostaghi and H. Azami, "Dispersion Entropy: A Measure for Time-Series Analysis," IEEE Signal Processing Letters, Vol. 23, No. 5, pp. 610-614, 2016

22. J. N. Xue and K. Yang, "Upper \& Lower Bounds of Stress-Strength Interference Reliability with Random Strength Degradation," IEEE Transaction on Reliability, Vol. 46, No. 1, pp. 142-145, 1997

23. B. Yeter, Y. Garbatov, and C. G. Soares, "Evaluation of Fatigue Damage Model Predictions for Fixed Offshore Wind Turbine Support Structures," International Journal of Fatigue, Vol. 87, No. 3, pp. 71-80, 2016

24. K. S. Zhang, J. G. Lin, and P. R. Xu, "A New Class of Copulas Involved Geometric Distribution: Estimation and Applications," Insurance Mathematics \& Economics, Vol. 66, No. 4, pp. 1-10, 2016

25. M. Y. Tsai, P. S. Huang, C. H. Lin, C. T. Wu, and S. C. Hu, "Mechanical Design and Analysis of Direct-Plated-Copper Aluminum Nitride Substrates for Enhancing Thermal Reliability," Microelectronics Reliability, Vol. 55, No. 12, pp. 2589-2595, 2015

26. M. Zubair and Q. Amjad, "Calculation and Updating of Common Cause Failure Unavailability by using Alpha Factor Model," Annals of Nuclear Energy, Vol. 90, No. 4, pp. 106-114, 2016

27. P. Zeephongsekul, C. L. Jayasinghe, L. Fiondella, and V. Nagaraju, "Maximum-Likelihood Estimation of Parameters of NHPP Software Reliability Models using Expectation Conditional Maximization Algorithm," IEEE Transactions on Reliability, Vol. 65, No. 3, pp. 1571-1583, 2016

Bin Suo received his Ph.D. from China Academy of Engineering Physics in 2012. He is an associate research fellow in the Institute of Electronic Engineering at China Academy of Engineering Physics. His research interests are uncertain information processing and system reliability analysis and evaluation. 